\title{
Experiments with UAS Imagery for Automatic Modeling of Power Line 3D Geometry
}

\author{
G. Jóźków, B. Vander Jagt, C. Toth * \\ Department of Civil, Environmental and Geodetic Engineering, The Ohio State University, Columbus, USA - (jozkow.1, vander- \\ jagt.1, toth.2)@osu.edu
}

Commission I, ICWG I/V-B

KEY WORDS: UAS, Dense Matching, Transmission Lines, Modeling

\begin{abstract}
:
The ideal mapping technology for transmission line inspection is the airborne LiDAR executed from helicopter platforms. It allows for full 3D geometry extraction in highly automated manner. Large scale aerial images can be also used for this purpose, however, automation is possible only for finding transmission line positions (2D geometry), and the sag needs to be estimated manually. For longer lines, these techniques are less expensive than ground surveys, yet they are still expensive. UAS technology has the potential to reduce these costs, especially if using inexpensive platforms with consumer grade cameras. This study investigates the potential of using high resolution UAS imagery for automatic modeling of transmission line 3D geometry.

The key point of this experiment was to employ dense matching algorithms to appropriately acquired UAS images to have points created also on wires. This allowed to model the 3D geometry of transmission lines similarly to LiDAR acquired point clouds. Results showed that the transmission line modeling is possible with a high internal accuracy for both, horizontal and vertical directions, even when wires were represented by a partial (sparse) point cloud.
\end{abstract}

\section{INTRODUCTION}

\subsection{Transmission Line Inspection}

Transmission line inspection provides important information for the utility service industry that improves asset management and risk mitigation. Specifically, it can be used to identify vegetation encroachment, wire height above ground, and changes in conductor parameters. The latter may result in overheating and consequently in changes of the wire sag (Polevoy, 1998). The low wire sag may indicate not only the need for conductor replacement, but may cause wire sag to dangerous levels. For these reasons, three-dimensional inspection of transmission line wires is both needed and conducted on a regular basis.

Corridor mapping employing remote sensing technologies is very often used to inspect transmission lines (Reed et al., 1996). Helicopter-based airborne LiDAR surveys seem to be the mostwidely used technology for monitoring power lines. Data collection is fast and modeling in all the three dimensions is automatic (Jwa et al., 2009). Airborne imagery has also been previously exploited for transmission line modeling, but was only used to extract the horizontal location of wires (Yan et al., 2007). There are other unconventional approaches to find the 2D geometry of power lines, such as airborne Synthetic Aperture Radar (Sarabandi and Park, 2003), but they are not commonly used in practice. All the above-mentioned techniques are generally characterized by high cost of data acquisition.

The cost of transmission line mapping by conventional airborne systems can be reduced by using UAS platforms with LiDAR sensors (Kuhnert and Kuhnert, 2013) or cameras (Li et al., 2010). The first approach shows better results, though, the absolute accuracy of the data may be low $(\sim 0.5 \mathrm{~m})$ in some cases. In addition, the price of LiDAR sensors is still higher than for cameras. The second approach requires relatively inexpensive equipment, but the modeling is limited to the horizontal position only.

This work shows the potential of utilizing UAS-imagery, acquired by commercial-off-the-shelf (COTS) cameras, for automatic modeling of transmission lines. In contrast to mentioned previous 2D techniques based on aerial imagery, the approach proposed in this paper allows for complete modeling of the 3D geometry. Since it uses only a medium grade commercial digital camera, the equipment cost is significantly lower compared to UAS LiDAR solutions.

\subsection{Potential of UAS Imagery}

The potential for direct transmission line modeling via photogrammetric techniques exists because of the high resolution of images, shown in Figure 1, captured at low altitudes by UAS platforms. Wires shown in such images can be seen and considered as small surface patches; albeit narrow and long. By applying dense matching algorithms after the images have been registered via bundle adjustment, direct surface reconstruction is generally feasible. The result of the dense matching is a point cloud and, consequently, could be used similarly to conventional LiDAR applications, such as modeling transmission line geometry. However, the quality and accuracy of the point cloud created by UAS imaging systems still need to be evaluated.

Many of the dense matching algorithms have been proposed in existing literature; e.g., a comparison of a few of them can be found in Ahmadabadian et al. (2013). Their groups vary significantly by underlying assumptions, range of use, and effects. The principles of some algorithms may differ only 
insignificantly, yet their use on the same data set may result in different point clouds. According to the best knowledge of the authors, there are no known algorithms that are dedicated to extracting thin elements similar to wires. This is likely due to the general constraints that are put into the algorithm and to the fact that ground and/or surface reconstruction is typically of the greatest importance. Points with large vertical offsets, such as between the wires and ground are likely to get filtered out as noise. Nevertheless, we believe that some of the existing algorithms can successfully match points on wires even if it requires appropriately taken images and its performance may be limited.

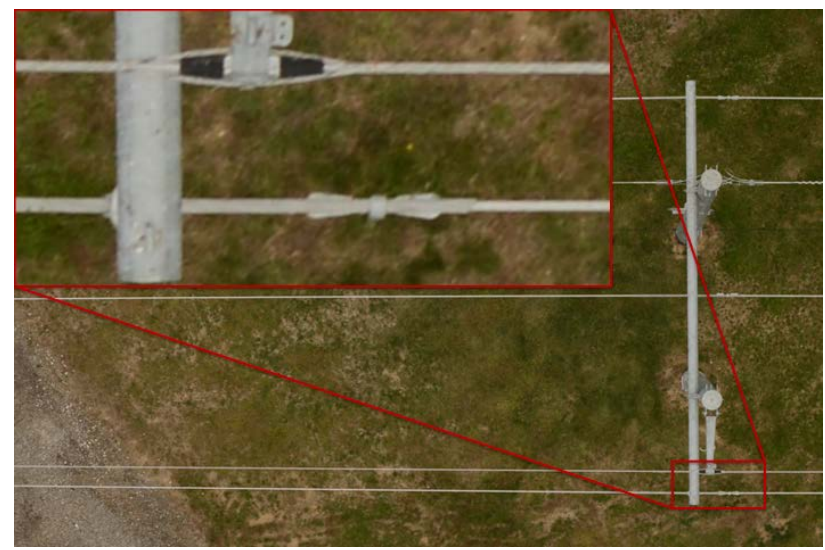

Figure 1. Example of the very high resolution UAS imagery taken over transmission line

\section{THE WORKFLOW OF MODELING TRANSMISSION LINE 3D GEOMETRY FROM UAS IMAGERY}

The proposed approach of transmission line modeling from UAS imagery consists of the following steps:

1. Data acquisition

2. Image block adjustment

3. Dense image matching

4. Point cloud filtering and segmentation

5. Transmission lines 3D geometry modeling

Detailed description of each step is given in subsequent sections.

\subsection{Data Acquisition}

There are several requirements of the imagery that are needed to be met in order to create points on wires. The scale of images should be large enough to represent the transmission wire with a few pixels across; preferably, showing the wire texture. Tests showed that the value of about 10 pixels is sufficient. The wire should be present in the depth of field, so the appropriate focusing and aperture number need to be set. The second requirement that should be considered is the image overlap. The higher the overlap (both end and side), the greater the reliability of the point reconstruction. Since the flying height cannot be too high, low flying speed is needed to maintain high endlap. Moreover, strips should be flown close to each other to assure high sidelap.

In addition to satisfying the imaging requirements, a georeferencing method to accurately orient images should be selected. This can be accomplished via the use of Ground Control Points (GCP) or accurate navigation data (GPS/IMU-based direct georeferencing).

\subsection{Image Processing}

In general, there are three methods for image orientation, namely the direct and indirect georeferencing approaches, and the combination of them, called integrated sensor orientation. To ensure the highest accuracy in this study, the indirect approach was used because of the large image scale and the effective use GCPs to constrain the adjustment. In this approach, the position and orientation of each image is determined globally by a bundle block adjustment (BA). An excellent survey of the photogrammetric bundle adjustment theory and its implementation aspects can be found in Triggs et al. (2010).

In normal airborne practice, the field measurement of GCPs is costly due to its time and labor requirements, and, therefore, the direct approach is generally preferred. In the direct approach, the camera positions and orientations are determined via differential observations from a multi-frequency GPS/GNSS receiver integrated with Inertial Measurement Unit (IMU) data. An interesting transition between classical direct and indirect georeferencing is the BA solution where instead of GCP coordinates accurate image positions are used to constrain the adjustment. This approach gives nearly identical accuracy as the BA based on GCPs (Jóźków et al., 2015, Vander Jagt et al., 2015).

Once the images are accurately georeferenced, a dense matching is performed and point cloud is created. In this work, we tested three different matching algorithms, implemented in commercial software. Since they are propriety, the details, obviously, are unknown to the user.

\subsection{Point Cloud Processing}

The processing of the point cloud to obtain the wire geometry consists of two main parts. First is the point cloud filtering, in which all points not belonging to the wires are removed. Second is the point cloud segmentation that arranges points into groups, resulting in one segment (group) for each wire. Since the main purpose of this experiment is to assess the feasibility of modeling the wire geometry based on UAS imagery, initially both tasks were performed manually. In the automatic approach, algorithms for filtering, such as presented by McLaughlin (2006), may be applied. The segmentation could be part of the modeling by using, for example, the RANSAC approach.

\subsection{D Modeling of Transmission Line Geometry}

Each transmission line can be geometrically described as a 2D curve, projection from the 3D space. Horizontally, it is a line:

$$
y=a \cdot x+b
$$

where $\quad a, b=$ parameters of the line

$x, y=$ horizontal coordinates

Vertically it is a catenary - a 2D curve describing wire sag:

$$
z=\cosh \left(\frac{u}{s}\right)
$$

where $\quad s=$ catenary parameter

$z=$ height

$u=$ point coordinates along wire (origin is at the center)

Since the basic equation of the catenary does not consider vertical and horizontal shifts, the lowest point always occurs for $u=0$ and has the height equal value of the parameter $s$, see Figure $2 \mathrm{~b}$. The 
vertical and horizontal shifts can be applied using two additional parameters $p, q$ in the basic catenary equation:

$$
z=s \cdot \cosh \left(\frac{u+p}{s}\right)+q
$$

Modeling the 3D geometry means finding all the five parameters $(a, b, p, q, s)$ describing the line and catenary. By fitting the line, Equation 1, to the point cloud segment with horizontal coordinates $x, y$, the two parameters $a, b$ can easily be found by least squares estimation. Fitting the catenary, however, is more complex. First, the horizontal coordinates $x, y$ need to be transformed according to wire distance $u$, see Figure 2a. This task can be solved in multiple ways; for example, by using azimuth estimated earlier of the line ( $a$ parameter):

$$
u=\frac{x}{\cos (\operatorname{atan} a)}
$$

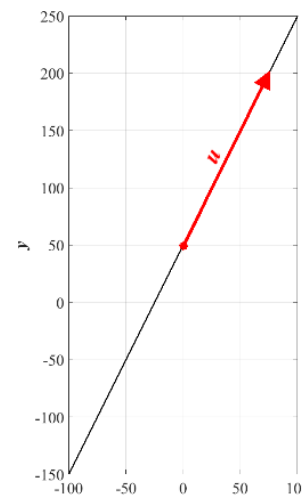

(a)

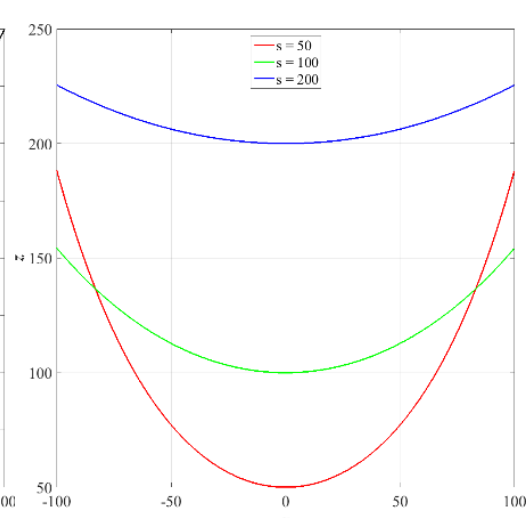

(b)
Figure 2. Geometry of the transmission line wire: (a) horizontal, (b) vertical; description in the text

Fitting the catenary with the help of the least squares method needs linearization of Equation 3, and consequently finding initial (approximated) values of the parameters $s, p, q$. In this work, the approximation of unknown parameters was found numerically based on three points using the LevenbergMarquardt method. These points were chosen from the point cloud segment as presented in Figure 3; two are placed on each end of the segment and the third one having the lowest height, preferably located in the middle of the sag.

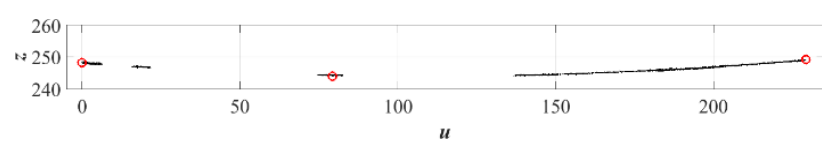

Figure 3. Location of points in the cloud segment to find initial catenary parameters

Both the line and the catenary fittings are done using noncontinuous wire segments, see Figure 3. Discontinuity or uneven point distribution along the curve can cause inappropriate fitting results when using the least squares method, particularly with respect to the catenary. This situation can be avoided if the geometry of modeled curve is known and/or appropriate initial parameters are chosen.

Because the least squares method is sensitive to outliers, robust estimation was applied to eliminate their influence. In particular, we utilized the Huber method (Huber, 1964). The (modified) Huber damping function changes weights of points $w$ in the next iteration depending on residuals $V$ from the previous iteration:

$$
w=\left\{\begin{array}{lc}
1, & \text { for }|V|<\sigma_{1} \\
\frac{|V|}{\sigma_{1}}, & \text { for } \sigma_{1} \leq|V| \leq \sigma_{2} \\
0, & \text { otherwise }
\end{array}\right.
$$

where $\sigma_{1}, \sigma_{2}$ are the Huber function parameters.

The parameters of the damping function decide on how the weights corresponding to different observations are modified. In particular, values of $5 \mathrm{~cm}$ and $50 \mathrm{~cm}$ were chosen as $\sigma_{1}$ and $\sigma_{2}$, respectively. Intuitively, this means that point observation which are within $5 \mathrm{~cm}$ (wire thickness) of the predicted fit are given a weight of 1 . Points observations in the range $5 \mathrm{~cm}$ to $50 \mathrm{~cm}$ are given lesser weights in the range of $1>w>0$, with the weight value being linearly dependent on the observation residual. Points with a residual larger than $50 \mathrm{~cm}$ are not considered in the fitting; weight equals 0 . The convergence threshold of the iterative process was set for $1 \mathrm{~mm}$ for all estimated parameters.

In this study, robust estimation could be applied because the point cloud was already filtered and segmented, and, therefore, larger number of inliers than outliers are present. For datasets without a priori manual segmentation, a RANSAC method could be applied, which is outside the scope of this research effort.

It should also be mentioned that the thickness of the wires was not modeled in this investigation, as it could potentially complicate the geometrical model, and the data (point cloud) is not accurate enough for this purpose.

\section{TEST DATA ACQUISITION}

\subsection{Equipment}

The flight to acquire data for this experiment was executed with the same equipment that was used in our previous studies (Jóźków et al., 2015). In short, the platform was a Bergen octocopter with a fixed nadir-viewing full frame 36 Mpix DSLR camera Nikon D800, see Figure 4. The camera was equipped with a constant focal length lens Nikon Nikkor AF-S $50 \mathrm{~mm}$ f/1.4G. Finally, a low cost GPS receiver, Solmeta Geotagger N3, was used to record the approximate position of each camera frame.

\subsection{Test Site and Flight}

The flight was executed for one section (separated by two towers) of a transmission line, which was approximately $230 \mathrm{~m}$ in length. The height of the towers was approximately $25 \mathrm{~m}$, see Figure 4 . The transmission line consisted of five wires placed at two levels; lower, L with 3 wires and upper, U with 2 wires, see Figures 4 and 5. Additionally, a sixth wire was present at the half of the pole height, see Figure 4, but the properties of the wire, such as thickness, mounting type, and insulation, confirmed that it was not a transmission line and, thus, it was not considered in the subsequent analysis.

The flight was planned at a height of $80 \mathrm{~m}$ above ground level and two overlapping strips were flown which were slightly longer than the length of the transmission line section, see Figure 6. The constant flying speed of $5 \mathrm{~m} / \mathrm{s}$ and $1 \mathrm{~Hz}$ of image acquisition rate resulted in more than $80 \%$ endlap for every part of the transmission line section, see Table 1 . Additionally, a second flight was flown with shortened strips and increased sidelap for testing purposes related to point cloud generation. 


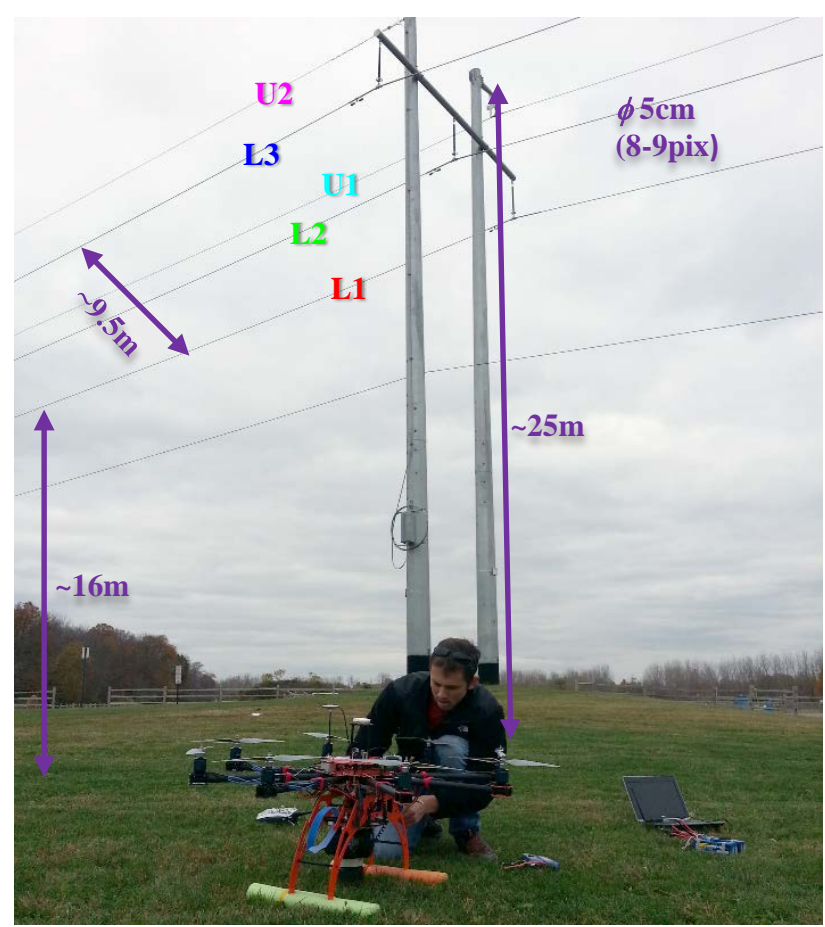

Figure 4. Part of the mapped transmission line section and used UAS

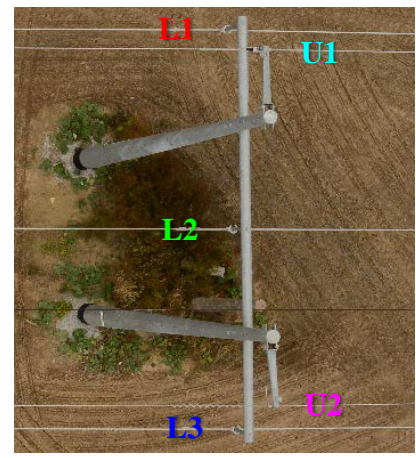

(a)

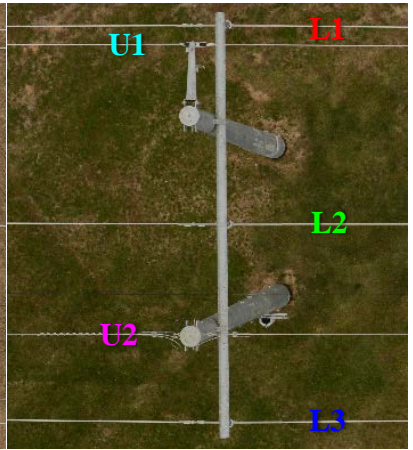

(b)
Figure 5. Top view on the towers at the end (a), and beginning (b) of the mapped transmission line section

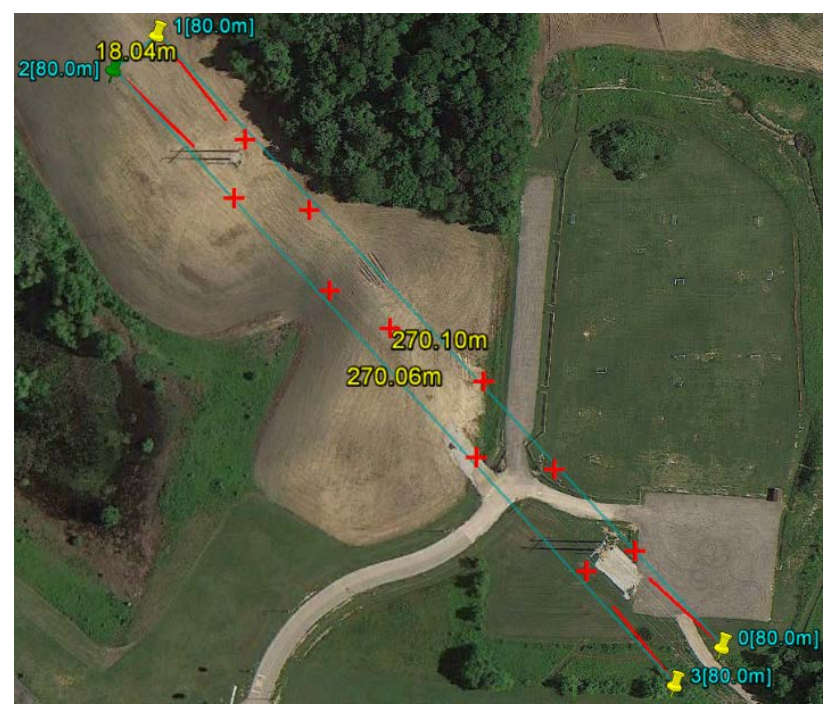

Figure 6. Flying plan of two strips and GCP locations

\begin{tabular}{|c|c|c|}
\hline Level & Ground & Wire \\
\hline GSD [mm] & 8 & $5-6$ \\
Endlap [\%] & 87 & $81-84$ \\
Sidelap [\%] & 69 & $55-61$ \\
\hline
\end{tabular}

Table 1. Parameters of images according to flight plan

The flight was executed in low wind conditions $(0-4 \mathrm{~m} / \mathrm{s})$. This ensured that the wires were stable during the image acquisition.

For increased georeferencing accuracy, 10 GCPs were signalized on the ground and measured using a dual frequency GPS receiver (RTK mode). The predicted accuracy of 3D position was $\sim 1.5$ $\mathrm{cm}$.

One aspect of using GPS and other sensor measurements in close proximity to transmission lines should be emphasized. The strong electromagnetic field may potentially be dangerous for the autopilot of the UAS, as many low cost systems utilize GPS and magnetometer observations to update the navigation solution, and both are susceptible to electromagnetic interference. In these experiments, no side effects were observed.

\section{RESULTS AND DISCUSSION}

\subsection{Image Block Adjustment}

The image bundle block adjustment was executed in several configurations that included different block configurations, three different software packages dedicated to UAS images and different processing options. Results of a few solutions are given in Table 2. Although the RMSE measured on GCPs was slightly different for different configurations, all had acceptable value for all the cases. Analyzing the obtained values, it can be seen that the third software provided the lowest errors on GCPs. Additionally, the block configuration of the single strip was adjusted with slightly lower accuracy.

\begin{tabular}{|c|c|c|c|c|}
\hline Solution & $\begin{array}{c}\text { Software } \\
\text { package }\end{array}$ & Images & Strips & $\begin{array}{c}\text { BA 3D } \\
\text { RMSE [cm] }\end{array}$ \\
\hline a & 1 & 200 & 2 long + 2 short & 3.0 \\
b & 1 & 62 & 1 long & 4.3 \\
c & 2 & 136 & 2 long & 3.5 \\
d & 3 & 136 & 2 long & 1.5 \\
e & 3 & 200 & 2 long + 2 short & 2.1 \\
\hline
\end{tabular}

Table 2. Sample results of image bundle block adjustment

\subsection{Point Cloud Generation}

The point clouds were generated using the same software packages in which the BA was performed. In this case, additional parameters were tested that may have impact on the dense matching results. Resulting point clouds that correspond to the presented in Table 2 solutions are shown in Figure 7. For the first software package, the configuration consisting from all strips and low density of generated points (Figure 7a), resulted in almost no points representing wires. Increasing the point density parameter, but running the same software for only one strip (Figure $7 \mathrm{~b}$ ), showed that some points were able to be matched at the wires, but they were outside the section chosen for the investigation. The likely reason was higher overlap at the ends of the strips where the vehicle slowed down and made turns while the frame rate of image acquisition remained the same. In this configuration, a larger noise in the point cloud was observed. Second software package was able to generate points for short parts of wires, but at many levels, which didn't clearly match with the wire structure (Figure $7 \mathrm{c}$ ). Therefore, without a priori 
knowledge of the geometry of the chosen transmission line section, subsequent processing would result in wrong number of detected wires. The last one tested software performed the best in terms of matching the points on wires. However, the point distribution was not even and points were not extracted for the entire wire lengths. Yet, clearly, these point clouds were the most complete among the tested configurations (Figure $7 \mathrm{~d}$ and 6e). For the subsequent processing, the last solution was selected (Figure 7e) since it had the largest number of points created for wires; though, it was the most noisy point cloud.

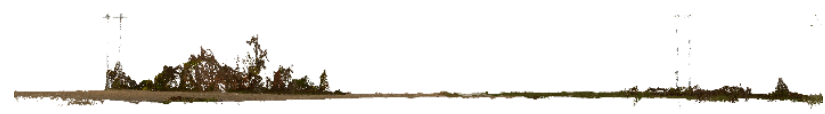

(a)

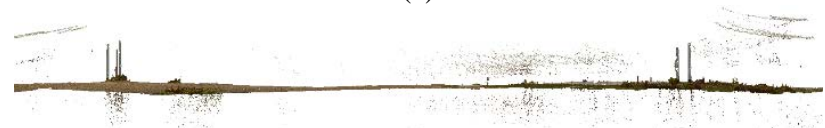

(b)

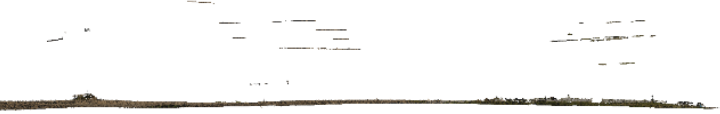

(c)

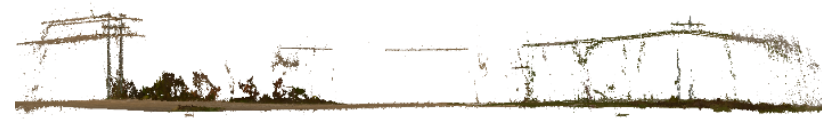

(d)

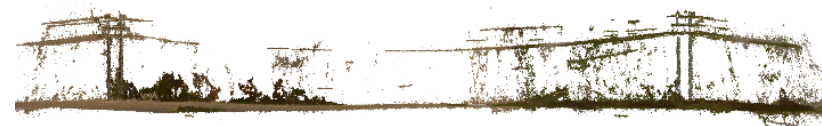

(e)

Figure 7. Examples of generated point clouds (side view) for various software packages and image block configurations

\subsection{Point Cloud Filtering and Segmentation}

As the main focus of this study is the feasibility of transmission line modeling, unwanted points were manually filtered, such as ground, vegetation, etc. Similarly, a point cloud segment for each individual wire (e.g., U1, L1, etc.) was also extracted manually. Since the transmission line components were well distinguished in the scene, the manual filtering was fast and easy. Figure 8 shows results after point segmentation where each point cloud segment, subjected for wire modeling, is distinguished by color, matching the color of the wire labels presented in Figures 4 and 5. Points belonging to other elements of the transmission line infrastructure, such as towers, wires outside chosen section, are shown in black. The number of points in each segment is shown in Table 3.
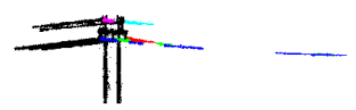

(a)

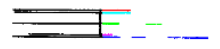

(b)

Figure 8. Filtered and segmented point cloud: (a) front view, (b) top view

\begin{tabular}{|l|c|c|c|c|c|}
\hline Wire & L1 & L2 & L3 & U1 & U2 \\
\hline Points & 46,021 & 25,432 & 35,247 & 17,415 & 3,259 \\
\hline
\end{tabular}

Table 3. Number of points segmented for each wire
Analyzing the number and point distribution over the wire, it can be seen that wires from the lower level $\mathrm{L}$ have the most complete reconstruction; note that there are still portions missing, e.g., in the middle and on the left side. Wires from the upper level $U$ are represented by much lower number of points. Each of the segments contain points at the end of wires that should assure good reconstruction in horizontal position. Note that the lack of points from the center portion of a line (e.g. U2) can cause difficulties in finding appropriate catenary parameters (Equation 3).

\subsection{D Modeling of Transmission Line Geometry}

The horizontal line reconstruction was successful for all wires, see Figure 9a. The internal accuracy of the horizontal line fit to the point cloud data was $8.5 \mathrm{~cm}$, see Table 4 . The estimated azimuth of the lines is nearly identical for lower level $\mathrm{L}$ lines and slightly different for upper line U1. The maximum azimuth difference of $0.4^{\circ}$ was observed for the U2 wire, but it was determined that this was due to a different mount (Figure 5) of this wire on the towers which resulted in the different azimuth value.

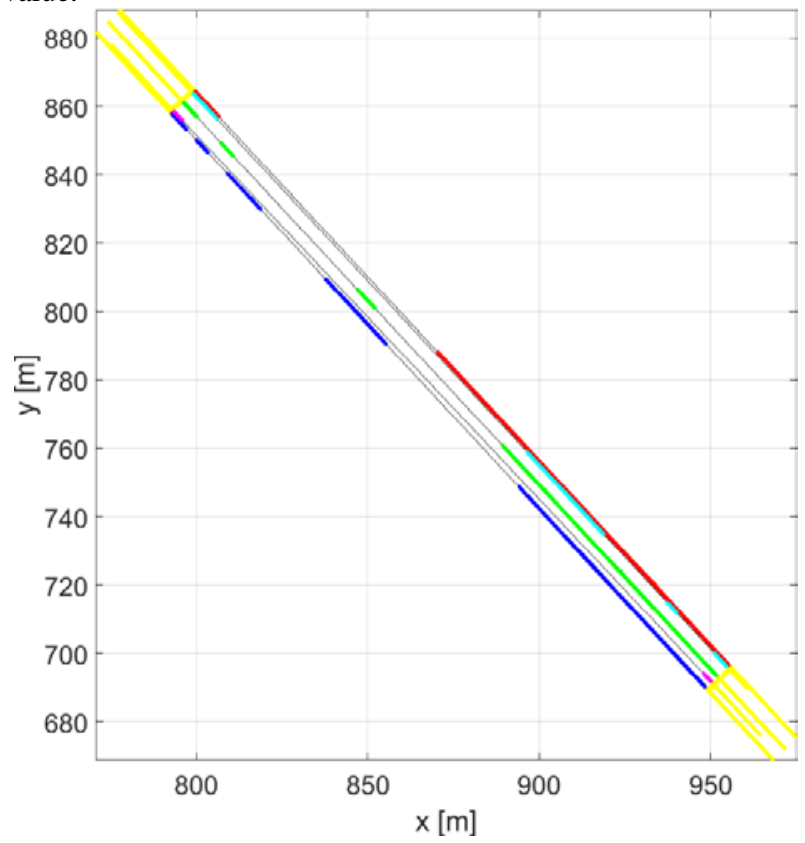

(a)

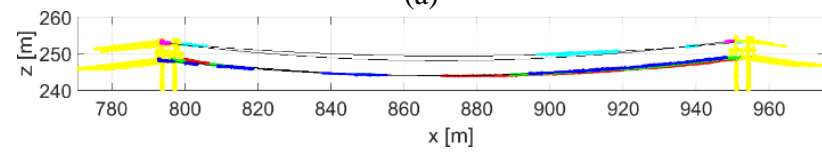

(b)

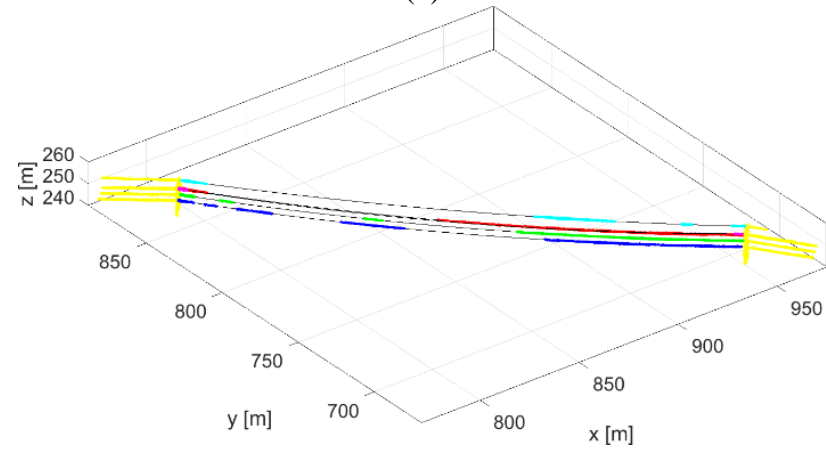

(c)

Figure 9. Wires reconstructed from estimated parameters: (a) top view, (b) side view, (c) isometric view 


\begin{tabular}{|c|c|c|c|c|c|}
\hline Wire & L1 & L2 & L3 & U1 & $\mathrm{U} 2$ \\
\hline $\begin{array}{l}\text { Horizontal } \\
\text { RMSE [cm] }\end{array}$ & 8.5 & 8.5 & 8.6 & 8.2 & 8.4 \\
\hline Azimuth $\left[^{\circ}\right]$ & -47.129 & -47.127 & -47.128 & -47.098 & -46.750 \\
\hline $\begin{array}{l}\text { Azimuth } \\
\text { RMSE [“] }\end{array}$ & 2.7 & 3.2 & 2.6 & 3.4 & 6.5 \\
\hline $\begin{array}{l}\text { Vertical } \\
\text { RMSE [cm] }\end{array}$ & 5.0 & 5.2 & 4.9 & 5.9 & 7.7 \\
\hline$s[\mathrm{~m}]$ & 1397.1 & 1413.2 & 1422.3 & 1789.7 & 1242.4 \\
\hline$s$ RMSE [m] & 0.24 & 0.35 & 0.28 & 0.90 & 23.21 \\
\hline
\end{tabular}

Table 4. Results of robust fitting of wires to the point cloud segments

The accuracy of fitting the catenary to the point cloud was also better than $10 \mathrm{~cm}$, see Table 4 . Analyzing the catenary parameter $s$, it can be seen that the lower level $\mathrm{L}$ wires have similar values with an estimated accuracy of $0.3 \mathrm{~m}$. The shape of the wire U1 is much different from the lower level $\mathrm{L}$ wires and the U2 line (Figure 9b) has the catenary accuracy three times worse than for the L lines. In the case of line U2, the sag accuracy is much lower than the others, confirming the lack of points in the middle section results in less reliable modeling.

\subsection{Relative Comparison of Catenary Shapes}

Since ground truth data was not available, validation of modeled wires was performed by comparing their shape. All catenaries should be identical if the wires are built from the same conductor, having the same length, and other parameters. Additionally, the relative height and distance between the start and end nodes should be the same for each wire.

The relative catenary comparison was based on assuming one of them as a reference and translating the other lines to the reference position; note that 3D translation vector and rotation in the horizontal plane were only considered. Comparison of height differences between these wires answers how similar each catenary is. The wire L1 was chosen as the reference since its parameter $s$ was estimated with the lowest RMSE, the points had most even distribution over the entirety of the line, and the overall amount of points was also the largest. Rotation of the other wires was based on the estimated azimuths, and the translation vector was calculated as the average between the corresponding two nodes of the reference and analyzed wire. The comparison was performed on the catenaries sampled with the distance of $1 \mathrm{~m}$; the smallest value that was found sufficient.

Results of the mean value of the vertical distance differences and RMSE are shown in Table 5. The shape of the lower level L lines is nearly identical; the error is in the range of the wire thickness and may be caused by modelling errors, but also by actual wire differences. Values obtained for the upper level wires show biases and an RMSE that is an order of magnitude higher than for the $\mathrm{L}$ lines. However, without ground truth data, it is impossible to say whether the reason is due to modeling errors or actual differences between wires, e.g. the length.

\begin{tabular}{|l|c|c|c|c|}
\hline Wire & L2 & L3 & U1 & U2 \\
\hline Mean [cm] & -3.6 & -5.5 & -68.4 & -38.9 \\
RMSE [cm] & 4.0 & 7.2 & 75.1 & 44.5 \\
\hline
\end{tabular}

Table 5. Results of relative catenary comparison to L1

\section{RECOMMENDATION FOR FUTURE}

The main recommendations to the future work coming from this experiment are related to the image parameters that allow for dense point matching on wires and their subsequent modeling. It seems that one of the key factors affecting numbers of matched points on the wire is the image overlap. By increasing both, sideand endlap, chances for better matching are higher. Obviously, the height of the flight cannot be too high since the number of pixels across the wire will be too low to present sufficient texture for the dense matching; note that a value around ten pixels seems to be a good choice for the given point cloud generating tools. Obviously, the flying height cannot be too low, as the wire could be too close to the UAS. In addition, the neighboring ground areas below the wires may have textures rapidly varying, making dense matching difficult. This effect was observed in the executed experiment on the wires from the upper level. They had lower number of points as well as lower reliability of the extracted parameters. Depending on the transmission line parameters, such as height and wire thickness, lenses with longer or shorter focal length may be used to compensate for changes in flying height.

In order to perform complete validation of the presented approach, a more comprehensive dataset is needed. In particular, transmission lines with differing design parameters in conjunction with ground truth data are necessary.

\section{CONCLUSIONS}

This work investigated the potential of using UAS imagery to model the 3D geometry of transmission lines. The experiments confirmed that line and catenary modeling based on the point cloud created by dense image matching is feasible, though, certain conditions need to be met. The most important is that the images should have very high overlap and sidelap as well as appropriate scale for both levels, ground and wires. This should be supplemented by a choice of appropriate dense matching algorithm allowing to extract points on the wires.

Initial results presented in this work showed internal modeling accuracy of around $8 \mathrm{~cm}$ and $6 \mathrm{~cm}$ for horizontal and vertical directions, respectively. The estimation of the wire parameters was possible even from the sparse data (the point cloud representing the wire only partially), but the reliability of the parameters was higher if the point cloud for the wire was more complete.

\section{REFERENCES}

Ahmadabadian, A. H., Robson, S., Boehm, J., Shortis, M., Wenzel, K., Fritsch, D., 2013. A comparison of dense matching algorithms for scaled surface reconstruction using stereo camera rigs. ISPRS Journal of Photogrammetry and Remote Sensing, 78, pp. 157-167.

Huber, P. J., 1964. Robust estimation of a location parameter. The Annals of Mathematical Statistics, 35(1), pp. 73-101.

Jóźków, G., Toth, C.K., Grejner-Brzezinska, D.A., 2015. On Sensor Georeferencing and Point Cloud Generation with sUAS. Proceedings of the ION 2015 Pacific PNT Meeting, Honolulu, Hawaii, April 20-23, pp. 839-848.

Jwa, Y., Sohn, G., Kim, H. B., 2009. Automatic 3D powerline reconstruction using airborne LiDAR data. The International Archives of the Photogrammetry, Remote Sensing and Spatial Information Sciences, XXXVIII-3/W8, pp. 105-110. 
The International Archives of the Photogrammetry, Remote Sensing and Spatial Information Sciences, Volume XL-1/W4, 2015 International Conference on Unmanned Aerial Vehicles in Geomatics, 30 Aug-02 Sep 2015, Toronto, Canada

Kuhnert, K. D., Kuhnert, L., 2013. Light-weight sensor package for precision 3D measurement with micro UAVs e.g. power-line monitoring. The International Archives of the Photogrammetry, Remote Sensing and Spatial Information Sciences, UAV-g2013, Rostock, XL-1 W, 2, pp. 235-240.

Li, Z., Liu, Y., Walker, R., Hayward, R., Zhang, J., 2010. Towards automatic power line detection for a UAV surveillance system using pulse coupled neural filter and an improved Hough transform. Machine Vision and Applications, 21(5), pp. 677-686.

McLaughlin, R., 2006. Extracting transmission lines from airborne LIDAR data. Geoscience and Remote Sensing Letters, IEEE, 3(2), pp. 222-226.
Sarabandi, K., \& Park, M., 2003. A radar cross-section model for power lines at millimeter-wave frequencies. IEEE Transactions on Antennas and Propagation, 51(9), pp. 2353-2360.

Triggs, B., McLauchlan, P. F., Hartley, R. I., Fitzgibbon, A. W., 2000. Bundle adjustment-a modern synthesis. In Vision algorithms: theory and practice. Springer Berlin Heidelberg, pp. 298-372.

Yan, G., Li, C., Zhou, G., Zhang, W., Li, X., 2007. Automatic extraction of power lines from aerial images. IEEE Geoscience and Remote Sensing Letters, 4(3), 387-391. 